\title{
Algunos LÍQuenes INTERESANTES DE El Teleno (LeÓn, NW de EsPaña)
}

\author{
ArsenioTERRÓN ALFONSO \& Leopoldo GARCÍA SANCHO
}

\begin{abstract}
RESUMEN.- El Teleno es un monte situado en la Sierra del mismo nombre, con una altitud de 2185 m., y que forma parte de los Montes de León. Destacan en él, los sustratos ácidos (cuarcitas, pizarras y areniscas). De los líquenes saxícolas que colonizan estos sustratos en alturas superiores a los $1700 \mathrm{~m}$., realizamos un comentario de aquellos más interesantes, destacando entre otros: Chrysotrix chlorina, Fuscidea intercincta, Lecidea furvella, Schaereria tenebrosa, Bellemerea alpina, Lecanora frustulosa, Orphniospora moriopsis var. bahusiensis, Rhizocarpon ferax, Rhizocarpon hochstetteri, Umbilicaria freyi y Umbilicaria hyperborea.
\end{abstract}

Palabras clave: Líquenes, saxícolas, NO de España.

SUMMARY.- El Teleno is a mountain placed in the mountain-chain with the same name. It is 2185 meters high and is a part of the Montes de León. It has acid substrata (quartzites,slates and sandstones). It is made a commentary of the saxicolous lichens living on those substrata over 1700 m., emphasizing among others: Chrysotrix chlorina, Fuscidea intercincta, Lecidea furvella, Schaereria tenebrosa, Bellemerea alpina, Lecanora frustulosa, Orphniospora moriopsis var. bahusiensis, Rhizocarpon ferax, Rhizocarpon hochstetteri, Umbilicaria freyi y Umbilicaria hyperborea.

Key Words: Lichens, saxicolous, NW of Spain.

\section{INTRODUCCIÓN}

El pico del Teleno se sitúa en el extremo suroccidental del arco formado por el extremo occidental de la Cordillera Cantábrica, Montes Astur-Galaicos y Montes de León, de estos últimos, El Teleno es el de máxima altitud con 2185 m. (29TQG). Nosotros analizamos la flora liquénica saxícola silicícola presente en el mismo, en alturas superiores a los $1700 \mathrm{~m}$.

Geológicamente se sitúa en el Dominio del Sinclinorio de Truchas, formado por un conjunto de materiales que siguen la dirección hercínica. En este Dominio, destaca el Anticlinorio del Teleno, estructura de gran envergadura, formada básicamente por una serie de pliegues similares bastante apretados. En el núcleo de este anticlinorio aflora la cuarcita del Argeriense que es una potente sucesión de cuarcitas blancas muy 
recristalizadas de gran dureza. Estas se disponen en sustratos muy potentes, separados por niveles de pizarras lustrosas y areniscas. La potencia de estos estratos varía de forma considerable entre el flanco norte y sur del anticlinorio, situándose en la zona de transición un estrato de areniscas ferruginosas, especialmente en la ladera sur (Velando \& al., 1981; Martínez \& al., 1981).

Debido a la falta de datos meteorológicos de las cumbres del Teleno nos es difícil la asignación del macroclima de esta zona, si bien, con la información que nos proporcionan las estaciones pluviotermométricas cercanas, puede apreciarse que el territorio soporta un clima fuertemente continental, con precipitaciones anuales que superan los $1000 \mathrm{~mm}$., siendo en muchas ocasiones en forma de nieve, que permanece en las partes culminales hasta bien entrada la primavera e incluso hasta el verano. La acción de los vientos húmedos, procedentes generalmente del oeste, provoca frecuentemente la existencia de nieblas, y muy especialmente la presencia de casquetes nubosos en altitudes superiores a los 1700-1800 m.

Biogeográficamente se enmarca en el subsector Maragato-Sanabriense (sector Orensano-Sanabriense, provincia Carpetano-Ibérico-Leonesa, región Mediterránea) (Rivas-Martínez, 1987), centrándose nuestro estudio en los pisos bioclimáticos supramediterráneo superior y oromediterráneo, con ombroclimas que van desde el húmedo medio al hiperhúmedo (Penas \& al., 1989). La vegetación superior que alberga este territorio es la referida a las siguientes series de vegetación Genisto sanabriensis-Junipereto nanae S., Saxifrago sphatularidi-Betuleto celtibericae S. y Holco molli-Querceto pyrenaicae S. (Penas \& al., 1989).

Los resultados que aquí reflejamos son un estracto del catálogo florístico del Teleno, actualmente en curso de elaboración. Para la elección de las especies que mencionamos a continuación se ha tenido en cuenta sobre todo su interés biogeográfico y la escasez de citas en la Península Ibérica. El material estudiado se encuentra depositado en el herbario LEB-Lich de la Universidad de León.

Acarospora oxytona (Ach.) Massal.

Presenta lóbulos con rugosidades o punteaduras, ascocarpos plano convexos, más oscuros que el talo, con borde talino persistente, lo que nos permite, de acuerdo con Poelt (1969: 84), Poelt \& Vezda (1977: 5) y Clauzade \& Roux (1981: 57 y 1985: 131), diferenciarla claramente de Acarospora chlorophana (Wahlenb.) Massal. Nuestros ejemplares muestran reacción negativa tanto en el talo como en la médula a todos los reactivos. Normalmente sobre cuarcitas recristalizadas muy duras, en extraplomos o paredes protegidas de la lluvia. En montañas y regiones frías de Europa, Asia y América del Norte (Clauzade \& Roux, 1981). Representada en toda la Península Ibérica.

\section{Alectoria sarmentosa (Ach.) Ach. subsp. sarmentosa}

Suele ocupar las grietas donde se produce un mayor acúmulo de suelo y humedad, acompañada generalmente por Sphaerophorus globosus. También con frecuencia tapiza paredes verticales junto a Bryoriafuscescens y Parmelia omphalodes. En Europa habitualmente epífita, en bosques de tipo boreal o subalpino (Hawksworth, 1972; Wirth, 1980). Muy rara en la zona 
ibérica occidental, donde hasta ahora sólo se conocía una cita en el macizo occidental de la Sierra de Gredos (Sancho, 1986: 62).

\section{Bellemerea alpina (Sommerf.) Clauz. \& Roux}

Ascocarpos criptolecanorinos, ligeramente plano-convexos, de color negro mate, sin tintes rojizos en ninguno de nuestros ejemplares. Reacciones: Talo $\mathrm{K}^{+}$(amarillo que pasa a rojo), $\mathrm{Pd}^{+}$(amarillo), médula $\mathrm{I}^{+}$(azul intenso). Epihimenio verdoso de 20-24 $\mu \mathrm{m}$ que da reacción $\mathrm{N}^{+}$(rojo-vinoso). Ascosporas de 9-12 (14) x 5.5-8 $\mu \mathrm{m}$.

Preferentemente sobre cuarcitas muy compactas, en situaciones muy expuestas tanto a la luz como al agua. Se trata de una especie de carácter alpino, típica de zonas montañosas con fuertes precipitaciones y abundante acumulación de nieve (Wirth, 1980; Creveld, 1981). En la Península Ibérica conocida de Aragón (Llimona, 1976), Pico del Lobo (Segovia) (Rico, 1983) y Sistema Central (Sancho, 1986).

\section{Cetraria commixta (Nyl.) Th. Fr.}

Médula con reacción negativa a todos los reactivos. Frecuente en las zonas más elevadas tanto sobre areniscas como cuarcitas, en grietas donde se acumula suelo, normalmente en situaciones protegidas del viento. Parece ser un taxon abundante en el centro y noroeste peninsular (Asturias; Vazquez, 1978), (Segovia; Rico, 1983), (Sistema Central; Sancho, 1986). En el centro y norte de Europa es bastante rara (Creveld, 1981; Wirth, 1980).

\section{Chrysotrix chlorina (Ach.) Laundon}

Talo de aspecto leprarioide, pero que en nuestros ejemplares presenta a la lupa (10X) un tipo de organización talina de aspecto pseudofruticuloso, de color marillo vivo, de 3-4 mm. de altura y formando talos de gran tamaño $(-15 \mathrm{~cm}$.). Coloniza cuarcitas muy duras y poco alteradas, en posiciones extraplomadas, en compañía de Acarospora chlorophana y Lecanora swartzii, o en posiciones verticales formando parte de comunidades muy llamativas junto con otros líquenes oscuros como Bryoria fuscescens, Parmelia omphalodes y Cornicularia normoerica. Hasta el piso altimontano, en montañas con grandes precipitaciones, borealsubmediterráneo (Wirth, 1980). Conocemos su presencia en el Sistema Central únicamente (Sancho, 1986).

\section{Fuscidea intercincta (Nyl.) Poelt}

Ascocarpos al principio con aspecto aspicilioide, luego ligeramente convexos, con disco negro, de 0.3-0.7 mm. Epihimenio de 20-25 $\mu \mathrm{m}$ ocre-marrón, himenio 50-60 $\mu \mathrm{m}$ hialino, hipotecio grisáceo con profusión de algas. Paráfisis poco coherentes, con la parte final inflada y marronácea. Ascos con dos capas amiloides muy marcadas, siendo la exterior muy patente (Sancho, 1986: 109). Ascosporas elipsoidales, de 11-14 x (4) 6-8 $\mu \mathrm{m}$, hialinas. Reacciones: Talo K, C, y KC negativo, médula I- Aparece en nuestro territorio sobre cuarcitas muy duras, en posiciones verticales o extraplomadas. En la Península Ibérica sólo era conocida hasta ahora de dos localidades: Serra da Estrella (Oberhollenzer \& Wirth, 1985) y Sierra de Guadarrama (Sancho, 1986).

\section{Hypogymnia farinacea Zopf}

Talo foliáceo de 2-3 $\mathrm{mm}$ de diámetro, grisáceo-verdusco, con pequeños isidios maciformes que se transforman en soralios de aspecto farináceo. Nuestros ejemplares muestran una reacción débilmente + con Pd en médula y fuerte en córtex superior (amarillo), 
lo que parece concordar con la raza química mencionada por Rico (1989: 164) sobre rocas ácidas. Sobre pizarras, en posiciones verticales, generalmente expuesta. De montana a subalpina (Wirth, 1980). No se conocía para el noroeste peninsular.

\section{Lasallia hispanica (Frey) Sancho \& Crespo}

Talo foliáceo-umbilicado, monófilo, rara vez polífilo, de $2-7 \mathrm{~cm}$., con pústulas dispersas. Superficie superior areolado-equinada, gris claro o blanquecina (aspecto de pequeños cristales), superficie inferior marronácea, en ocasiones débilmente decolorada, lisa o ligeramente areolada. Presenta siempre isidios escuamiformes, preferentemente en el borde del talo. Ascocarpos abundantes, ligeramente estipitados, negro mate, de $2-4 \mathrm{~cm}$., superlecideinos. Epihimenio 43-47 $\mu \mathrm{m}$ marrón, himenio 140-160 (200) $\mu \mathrm{m}$ hialino, subhimenio 120-130 $\mu \mathrm{m}$ ocre pálido, hipotecio 80-100 $\mu$ m gris-marrón. Esporas 48-52 (56) x 20-24 $\mu \mathrm{m}$ x 2 fuertemente murales, ocráceas, a veces hialinas. Taxon mediterráneo occidental, especialmente abundante en las zonas montañosas del Escudo Hespérico (Sancho \& Crespo, 1989).

\section{Lecanora concolor Räs.}

Ascocarpos ligeramente levantados sobre las areolas, con reborde patente y al igual que el disco concóloro al talo, de 0.5-1.2 mm. Epihimenio de 20-25 $\mu \mathrm{m}$ marronáceo-verdoso. Ascosporas de 7-9 (10) x 4-5.5 (6.5) $\mu \mathrm{m}$, hialinas. Conidios ligeramente curvados, de 15-25 x $1 \mu \mathrm{m}$. Lo hemos encontrado en una sola ocasión, sobre cuarcita, en compañía de Letharia vulpina, lo que contrasta con la abundancia de la misma en el Sistema Central (Sancho, 1986: 146). Parece tener una preferencia oromediterránea s. l. occidental y más concretamente por los macizos occidentales del Sistema Central (Sancho, 1986).

\section{Lecanora frustulosa (Dicks.) Ach.}

Talo crustáceo-areolado, de 10-15 cm., de color verde-limón, formado por areolas sumamente pequeñas (0.2-0.5 mm.), rugulosas, sin hipotalo patente. Ascocarpos muy abundantes, de sentados a subestipitados, planos o ligeramente convexos, desde líbidos hasta negros, en ocasiones recubiertos de ligera pruina, de $0.5-1 \mathrm{~mm}$., con borde regular o débilmente sinuoso. Epihimenio de 20-23 $\mu \mathrm{m}$ marronáceo. Ascosporas de 9-11 x 5-6 $\mu \mathrm{m}$, hialinas. Ascos con tolus amiloide típico (Hafellner, 1984). Reacciones: Talo $\mathrm{K}^{+}$amarillento, $\mathrm{C}^{-}, \mathrm{KC}^{+}$amarillo, $\mathrm{Pd}^{+}$anaranjado. Disco del ascocarpo $\mathrm{Pd}^{-}$, borde $\mathrm{Pd}^{+}$naranja-ferruginoso. Taxon ártico-alpino, sólo citado hasta ahora en España para las altas montañas almerienses (Egea, 1980).

\section{Lecanora swartzii (Ach.) Ach.}

Talo bulado, verrucoso a subfruticuloso, de color grisáceo con ligeros tintes amarillentos, de 4-5 cm., con hipotalo blanco muy patente en la periferia. Ascocarpos muy abundantes, cubriendo casi todo el talo, subestipitados, de plano a cóncavos, con borde muy patente, disco liso, pruinoso, $\mathrm{C}^{+}$amarillo-naranja, de 0.9-1.6 mm. Epihimenio de 18-22 $\mu \mathrm{m}$ marronáceo, himenio 60-70 $\mu \mathrm{m}$ hialino. Ascosporas subglobulosas de 8-10 x 6-7 $\mu \mathrm{m}$ hialinas X 8. Ascos con tolus amiloide típico (Hafellner, 1984). Talo $\mathrm{K}^{+}$amarillo, $\mathrm{C}^{+}$amarillo. Aparece en nuestra zona en posiciones verticales o en semicuevas, generalmente muy protegido o en lugares con fuerte escorrentía, acompañando en ocasiones a Chysotrix chlorina. Seguimos en este caso el concepto nomenclatural de Nimis \& Poelt (1987), que consideran a Lecanora subradiosa como un sinónimo de esta especie. En montañas fuertemente oceánicas (Wirth, 1980) medioeuropeas y submediterráneas. Hasta el momento conocida solamente en el Sistema Central (Rico, 1983; Sancho, 1986). 
Lecanora sulphurea (Hoffm.) Ach.

Ascocarpos de color negro-azulado, imarginados en visión superficial, convexos y con contorno generalmente irregular, de tamaño que excede en ocasiones al de las areolas. Epihimenio verde-negruzco de $20-25 \mu \mathrm{m}, \mathrm{N}^{+}$rojo-vinoso. Ascosporas elipsoidales de (8) 10 14 (16) x (4) 5-8 (9) $\mu \mathrm{m}$, hialinas. El talo reacciona $\mathrm{K}^{+}$amarillo intenso pasando luego a marronáceo, $\mathrm{C}^{+}$ligeramente rosado y $\mathrm{KC}^{+}$amarillento. Aparece tanto sobre cuarcitas,como areniscas y pizarras, siendo abundante hasta $\operatorname{los} 2000 \mathrm{~m}$. de altitud, tanto en espolones como en paredes verticales o en grietas en las que se acumula materia orgánica. Abundante en el sur y sureste peninsular (Werner, 1979; Egea \& Llimona, 1979), y muy rara en el centro peninsular (Sancho, 1986).

\section{Lecidea furvella Nyl. ex Mudd.}

Talo crustáceo, marrón oscuro a negruzco, con la cara superior sorediado-granulosa, de $1-1.5 \mathrm{~cm}$. Ascocarpos sobre las areolas, negros planos y con pequeño reborde concoloro. Epihimenio 18-23 $\mu \mathrm{m}$ marronáceo, himenio 80-90 $\mu \mathrm{m}$ hialino-ocráceo, hipotecio y excípulo marrón oscuro, que reaccionan $\mathrm{K}^{+}$formando cristales rojos, $\mathrm{C}^{+}$rojo y $\mathrm{Pd}^{+}$naranja. Ascos sin tolus amiloide. Ascosporas elipsoidales, de (11) 12-14 x 6-7 $\mu$ m hialinas. Talo $\mathrm{K}^{+}$amarillo, $\mathrm{Pd}^{+}$amarillo-naranja, $\mathrm{C}^{-}$. Médula $\mathrm{I}^{-}$. Aparece en rocas cercanas al suelo, acompañado y semiprotegido por Umbilicaria polyphylla. En España sólo se la conocía anteriormente en el Sistema Central (Sancho, 1986).

\section{Orphniospora moriopsis (Massal.) Hawksw. var. bahusiensis (Degel) Poelt}

Areolas de 0.2-0.6 mm., convexas y lisas. Ascocarpos muy abundantes, con el disco verde oscuro hasta negro, con borde negro patente, de 0.4-1.2 mm. Excípulo carbonáceo, bien desarrollado, epihimenio de 20-24 $\mu$ m marronáceo, $\mathrm{K}^{+}$vinoso-rosado, himenio de 110 a 140 $\mu \mathrm{m}$ de hialino a ocre pálido. Ascos con tolus amiloide típico (Hafellner, 1984: 313). Aparece en nuestro territorio a partir de los $1850 \mathrm{~m}$. preferentemente sobre areniscas y cuarcitas muy duras, en espolones, cimas y paredones muy expuestos. Se cita por segunda vez para la Península Ibérica, anteriormente conocida del Sistema Central (Sancho, 1986).

\section{Porpidia crustulata (Ach.) Hertel \& Knoph.}

Talo con reacciones negativas al $\mathrm{K}, \mathrm{C}, \mathrm{KC}$, médula negativa al $\mathrm{K}, \mathrm{C}, \mathrm{KC}$ e $\mathrm{I}$. Ascocarpos muy abundantes, sentados, negros, con reborde concoloro y regular, de $0.8-1.3$ $\mathrm{mm}$., de planos a ligeramente convexos, brillantes y sin pruina. Excípulo ocráceo, $\mathrm{K}^{-}$, epihimenio de 30-35 $\mu \mathrm{m}$ verdoso, $\mathrm{N}^{+}$pardo-rojizo, himenio de $80-90 \mu \mathrm{m}$ hialino. Ascos con tolus amiloide reducido a dos bandas muy estrechas (Hafellner, 1984: 321). Esporas elipsoidales de (14) 16$18 \times 5-7(8) \mu \mathrm{m}$, hialinas. Lo hemos recolectado sobre arenisca, en posiciones cercanas al suelo y en lugares húmedos. Montano y boreal-mediterráneo (Wirth, 1980). En la Península Ibérica conocido de El Montseny (Hladun, 1982), Sevilla (Rowe \& Egea, 1985), Pico del Lobo (Rico, 1983) y Sistema Central (Sancho, 1986).

\section{Rhizocarpon ferax $\mathrm{H}$. Magn.}

Ascocarpos redondeados, planos, de menos de $1 \mathrm{~mm}$., generalmente rebordeados por las areolas. Epihimenio de 48-56 $\mu$ m ocráceo, $\mathrm{K}^{-}$, himenio de 200-225 $\mu \mathrm{m}$ hialino, en ocasiones con tintes verde-azulados, excípulo patente, carbonáceo. Ascos claviformes, con esporas murales de 34-40 (48) x 13-16 (20) $\mu \mathrm{m}$ de verde oscuro a marroneáceas. Reacciones: Talo $\mathrm{Pd}^{+}$ 
débilmente amarillento, $\mathrm{K}^{+}$amarillo, médula $\mathrm{K}^{-}, \mathrm{Pd}^{+}$amarillo, $\mathrm{I}^{+}$azul fuerte. Sólo aparece sobre las cuarcitas más duras. Especie de distribución ártico-circumpolar, especialmente abundante en Escandinavia (Runemark, 1956), en la Península Ibérica conocido de algunas localidades del Montseny (Hladun, 1982) y del Sistema Central (Sancho, 1986).

\section{Rhizocarpon hochstetteri (Körb.) Vain.}

Talo crustáceo-areolado, de color gris, a veces con tintes marrón-rojizo, con hipotalo negro muy patente. De $1-2.5 \mathrm{~cm}$., areolas pequeñas $(<0.5 \mathrm{~mm}$.). Ascocarpos de pequeño tamaño $(<0.5 \mathrm{~mm}$.) de color negro, insertos en las areolas, desde plano a ligeramente convexos. Epihimenio de 28-35 $\mu \mathrm{m}$ que reacciona $\mathrm{K}^{+}$amarillo-oro y $\mathrm{N}^{+}$rojo-sangre. Ascosporas de $13-$ 18 x 7-9 $\mu \mathrm{m}$, hialinas. Reacciones: Talo $\mathrm{K}^{+}$verde y luego ferruginoso, médula $\mathrm{C}^{-}, \mathrm{KC}^{+}, \mathrm{I}^{-}$. Presente preferentemente sobre rocas muy duras. Desde medianamente fotófilo a esciófilo. De submontano a montano (Feuerer, 1978; Wirth, 1980). No conocemos otras citas para España.

\section{Rhizocarpon polycarpum (Hepp.) Th. Fr.}

Ascocarpos planos a ligeramente convexos, con disco negro, de 0.5-1 mm. Excípulo estrecho, marrón negruzco, epihimenio de 23-28 $\mu \mathrm{m}$ marrón rojizo, $\mathrm{K}+$ débilmente rojo, himenio de 65-80 $\mu \mathrm{m}$ hialino, subhimenio de 16-20 $\mu \mathrm{m}$ ocráceo, hipotecio negruzco. Ascosporas 15-20 (24) x 8-1 (11) $\mu \mathrm{m}$, hialinas X 8. Reacciones: Talo $\mathrm{K}^{-}, \mathrm{C}^{-}, \mathrm{KC}^{-}$, médula $\mathrm{I}^{+}$ azul-negro. Aparece en areniscas ferruginosas, en las zonas más elevadas (>1900 m.). De montano a alpino (Feuerer, 1978). Sólo lo conocemos citado para el centro y suroeste peninsular (Rico, 1983; Sancho, 1986; Rowe, 1985).

\section{Rhizocarpum riparium Ras. subsp. riparium}

Ascocarpos grandes (de 0.7-1.3 mm.), ligeramente hundidos, con reborde blanquecino muy patente. Epihimenio de 18-22 $\mu \mathrm{m}$ verdoso, $\mathrm{K}^{-}$, himenio de $160-190 \mu \mathrm{m}$ verde esmeralda en la parte superior haciéndose hialino en la parte inferior, excípulo de tonos claros hacia el interior. Esporas grandes, de 40-48 x 16-20 $\mu \mathrm{m}$, verdosas, fuertemente murales. Reacciones: Talo $\mathrm{Pd}^{+}$amarillo-oro, médula $\mathrm{Pd}^{+}$amarillo, $\mathrm{I}^{+}$azul-negro. Recogido sobre cuarcitas, en situaciones expuestas. Conocido de Lérida (Runemark, 1956) y del Sistema Central (Sancho, 1986), otros autores lo citan sin asignar rango subespecífico (Rowe \& Egea, 1985).

Rhizocarpon superficiale (Schaer.) Vain subsp. superficiale

Talo crustáceo-areolado, de color amarillo intenso, de 7-10 cm., con hipotalo negro, patente sobre todo en el borde. Areolas grandes (0.6-1.4 mm.), generalmente escabrosashendidas. Como ya señaló Runemark (1956: 55), nuestros ejemplares parecen referirse a la transición que él señala para el oeste de la Península Ibérica entre la subsp. superficiale y la subsp. boreale, en lo referente al color y tamaño de las areolas. Talo $\mathrm{K}^{-}, \mathrm{Pd}^{-} \mathrm{o}^{+}$débilmente rojizo, médula $\mathrm{I}^{-}, \mathrm{Pd}^{-}, \mathrm{K}^{-}$. Ascocarpos abundantes, desde cóncavos a planos, papilosos, grandes (0.6-1 mm.), negros, mate, angulosos. Epihimenio de 20-24 $\mu \mathrm{m}$ marrón, $\mathrm{K}^{+}$rojizo, himenio 85-100 $\mu \mathrm{m}$ hialino, hipotecio $120-150 \mu \mathrm{m}$ ocre, $\mathrm{K}^{+}$amarillo. Esporas bicelulares, de 13-16 x (8) 10-12 $\mu \mathrm{m}$, marrón-oliváceas X 8. Solamente lo hemos localizado en la cumbre de El Teleno, sobre cuarcita y en posiciones muy expuestas.

\section{Schaereria tenebrosa (Flot.) Hertel \& Poelt}

Talo crustáceo-areolado, medianamente grueso, de color marronáceo, con hipotalo negro bien visible. Ascocarpos al principio algo hundidos, finalmente sentados, con el disco 
negro y con reborde propio, fino en los adultos, de 0.3-0.8 $\mathrm{mm}$. Excípulo carbonáceo en la parte más externa, grisáceo hacia el interior, epihimenio de 40-45 $\mu \mathrm{m}$ negro-verdoso, $\mathrm{N}^{+}$púrpura, himenio 68-75 $\mu \mathrm{m}$ de hialino a ligeramente violáceo, subhimenio 40-45 $\mu \mathrm{m}$ hialino, hipotecio 20-25 $\mu \mathrm{m}$ marronáceo. Ascos octosporados sin tolus amiloide. Ascosporas hialinas de 11-13 x 4.5-6 $\mu$ m elipsoidales. Si bien su óptimo corológico se centra en el área boreal (Wirth, 1980; Creveld, 1981), aparece con relativa frecuencia en nuestro territorio, principalmente sobre pizarras y areniscas. La confusión posible entre este taxon y Lecidea griseoatra (sin. p. p.) no permite asegurar las citas que corresponden a cada uno de ellos.

Sphaerophorus globosus (Huds.) Vain.

Si bien su comportamiento parece ser preferentemente cortícola, no es raro encontrarlo en nuestra zona, tanto en grietas donde se acumula materia orgánica, como en paredes más o menos verticales y expuestas en compañía de Parmelia omphalodes y Bryoria fuscescens.

Algunos de nuesstros ejemplares parecen encajar en la variedad curtus (Hook. \& Tayl.) Zahlbr. (Clauzade \& Roux, 1985: 710), de menor tamaño, en los que no hay diferenciación neta entre el tronco principal y las proliferaciones laterales. No se conocen citas de esta variedad para la Península.

\section{Umbilicaria hyperborea (Ach.) Hoffm.}

Talo foliáceo-umbilicado, de 3-5 cm., de color marrón oscuro en su cara superior y ligeramente brillante, con aspecto hendido-areolado, cara inferior negra, sin rizinomorfos, ligeramente areolada. Ascocarpos muy abundantes en todo el talo, tipo girodisco, negros, de 0.8-1.6 mm., ascosporas simples, de 11-13 x 8-9 $\mu$ m hialinas. La hemos localizado en un sólo punto, en la cima de El Teleno, en una superficie horizontal, sobre cuarcita. Representa un nuevo punto en la distribución de esta especie hacia el oeste de Europa, pues si bien su óptimo corológico parece situarse en Europa Central y del Norte, ya Poelt (1969: 679) la cita para el norte de Portugal. En España no había sido citada anteriormente, aunque conocíamos un pliego procedente del Puerto de Leitariegos (Asturias) (L. G. Sancho, 06-09-1980, MAF-Lich).

\section{Umbilicaria freyi Codogno, Poelt \& Puntillo}

Talo foliáceo-umbilicado, de 4-7 cm., cara superior gris-ceniza, débilmente areolada y con esquizidios abundantes y muy patentes (Codogno \& al., 1989: 63), especialmente en la periferia. Cara inferior pálida, ligeramente ocrácea, areolada, con escasos rizinomorfos, más concentrados en la periferia. No hemos encontrado ejemplares fructificados. Presente tanto en cuarcitas como en pizarras, en una banda altitudinal amplia. Muy abundante en todo el territorio, ampliando así su áreal hacia el oeste pues hasta el momento el punto más occidental que se conocía se refiere a un ejemplar recogido por nosotros en el año 1985 en Cardaño de Arriba (Palencia) (Codogno \& al., 1989: 66). Generalmente aparece en posiciones verticales e incluso extraplomadas.

\section{BIBLIOGRAFÍA}

CLAUZADE, G. \& C. ROUX -1984- Les genres Aspicilia Massal. et Bellemerea Hafellner et Roux. Bull. Soc. Bot. Centre-Ouest, nov. ser., 15: 127-141.

CLAUZADE, G. \& C. ROUX -1985- Likenoj de Okcidenta Europo. Bull. Soc. Bot. du CentreOuest. Nouvelle série № Special: 7.

CODOGNO, M., J. POELT \& D. PUNTILLO -1989- Umbilicaria freyi spec. nova und der Formenkreis von Umbilicaria hirsuta in Europa (Lichenes, Umbilicariaceae). Pl. Syst. Evol., 165: 55-69. 
CREVELD, M. -1981- Epilithie Lichen communities in the Alpine zone of Sourthern Norway. J. Cramer, F1-9490 Vaduz.

EGEA, J.M. -1980- Flora y vegetación de los líquenes de rocas silíceas no volcánicas del SE de España. Memoria Doctoral. Murcia.

EGEA, J.M. -1985- Algunos líquenes silicícolas interesantes de la Sierra de Los Filabres (Almería) y Sierra Nevada (Granada). España. Act. Bot. Malac., 10: 3-10.

FEUERER, T. -1978-Zur Kenntnis der Flechtengattung Rhizocarpon in Bayer. Ber.Bayer. Bot. Ges., 49: 59-135.

HAFELLNER, J. - 1984- Studien in Richtung einer natürlichen Gliederung der Sammelfamilien Lecanoraceae und Lecideaceae, pp. 241-371. In H. Hertel \& F. Oberwinkler (eds.), Beitrage zur Lichenologie. Festscrift J. Poelt. Beiheft zur nova Hedwigia 79. J. Cramer, Vaduz.

HERTEL, H. - 1984- Uber saxicole, lecideoide Flechten der subantarktis. pp. 399-499. In H. Hertel \& F. Oberwinkler (eds.), Beitrage zur Lichenologie. Festscrift J. Poelt. Beiheft zur nova Hedwigia 79. J. Cramer, Vaduz.

HLADUN, N.L. -1982- Aportación a la flora, morfología y vegetación de los líquenes silicícolas de la parte alta de Montseny (Cataluña). Memoria Doctoral.

LLIMONA, X. -1976-Prospecciones liquenológicas en el Alto Aragón Occidental. Collectanea Bot. Vol. 10 n 12 .

MARTÍNEZ, F.J., F. VELANDO \& J. MATAS -1982- Mapa Geológico de España. Escala 1: 50.000. Hoja 230: Castrocontrigo. I.G.M.E. Ministerio de Industria y Energía.

NIMIS, P.L. \& J. POELT -1987- The lichens and lichenicolous fungi of Sardinia (Italy). Studia Geobotánica, 7 (1).

PENAS, A., E. PUENTE, C. PÉREZ, Mà.J. LOPEZ, L. HERRERO \& M.E. GARCÍA -1989Mapa ombroclimático de la provincia de León. Escala 1: 200.000. Inédito.

POELT, J. -1969- Bestimmungschlüssel europaischer Flechten. Lehre.

POELT, J.\& A. VEZDA -1977-Bestimmungschlüssel europaischer Flechten. Erganzungsheft I. Bibli. Lich. (Vaduz), 16.

POELT, J.\& A. VEZDA -1981-Bestimmungschlüssel europaischer Flechten. Erganzungsheft II. Bibli. Lich. (Vaduz), 16.

RICO, V. -1983-Líquenes de los pisos oro- y crioromediterráneos del Pico del Lobo (Sierra de Ayllón, Segovia). Mem. Licenciatura.

RICO, V. -1989-Líquenes de rocas silíceas de los pisos meso- y supramediterráneos de la provincia de Madrid (España). Mem. Doctoral.

RIVAS-MARTÍNEZ, S. -1987- Memoria del Mapa de Series de Vegetación de España. Escala 1: 400.000. ICONA. Ministerio de Agricultura, Pesca y Alimentación.

ROWE, J. \& J.M. EGEA -1985- Contribución al estudio liquenológico del Sur de España, I: Líquenes silicícolas de la zona más térmica de la provincia de Sevilla. Lazaroa 8:333-352.

ROWE, J. \& J.M. EGEA -1987- Líquenes silicícolas de Sierra Morena, I. Act. Bot. Malc. 12: 59-66.

RUNEMARK, H. -1956-Studies in Rhizocarpon I: Taxonomy of the yellow species in Europe. Opera Bot. 2 (1): 1-152.

SANCHO, L.G. - 1982- Nuevos datos para las Umbilicariaceae (Líquenes) ibéricas. Collec. Bot. 13 (1): 339-349.

SANCHO, L.G. -1986- Flora y vegetación liquénica saxícola de los pisos oro-y crioromediterráneo del Sistema Central español. Memoria Doctoral.

SANCHO, L.G. \& A. CRESPO - 1989- Lasallia hispanica and related species. Lichenologist $21(1): 45-58$.

VÁZQUEZ, V.M. -1978- Notas liquenológicas I. Aportaciones al catálogo asturiano. Rev. Fac. Cienc. Univ. Oviedo, 17-19: 295-301.

VELANDO, F., J. MATAS \& A. PÉREZ -1981- Mapa geológico de España. Escala 1: 50.000. Hoja 192: Lucillo. I.G.M.E. Ministerio de Industria y Energía.

WERNER, R.G. -1979- La flore lichenique de la Cordillera Bético-Rifaine. Collec. Bot. Vol $9, \mathrm{n}^{\mathrm{o}} 17$.

(Aceptado para su publicación en Junio de 1.990)

Dirección de los autores: A. Terrón Alfonso, Dpto. Biología Vegetal. Universidad de León.

L. García Sancho, Dpto. Biología Vegetal. Universidad Complutense. 